\title{
Probing the Electric-Field Modulated Ferroelectric Liquid Crystal Films with Phase-Sensitive Raman Imaging Technique
}

\author{
Yun H. Wang and Jung Y. Huang ${ }^{*}$ \\ Department of Photonics and Institute of Electro-Optical Engineering, Chiao Tung University, Hsinchu, Taiwan, \\ Republic of China
}

\begin{abstract}
The electric-field-induced modulation of surface stabilized ferroelectric liquid crystal (SSFLC) films was investigated with a phase sensitive Raman imaging. We found the technique not only effectively suppresses the nonresonant background of a Raman spectrum, but also yields an insight into the modulated amplitudes and phases of Raman peaks. By using the technique, the electro-optical switching SSFLC film doped with $\mathrm{ZnO}$ nanocrystals ( $n c$ - ZnO) was shown to switch in a more organized way and be less sensitive to the modulation induced disordering than an undoped SSFLC film. The method can be implemented in a conventional Raman spectroscopy to allow researchers probing into the spatial distribution and modulation dynamics of electro-optic active species in a complex thin film.
\end{abstract}

PACS: $300.6450 ; 180.0180 ; 160.3710 ; 170.4090$.

\section{INTRODUCTION}

Raman scattering belongs to an inelastic light scattering which creates or annihilates an optical phonon in a medium. When a photon bounces off a molecule, the inelastic scattered photon is less energetic and the scattered photon exhibits a frequency shift. The various frequency shifts associated with different molecular vibrations give rise to a spectrum, which is characteristic of a specific compound. Therefore, Raman spectroscopy has been widely used as a molecular finger-printing probe to identify molecular species and structures of a complex material. Unenhanced and nonresonant Raman signal is extremely weak and often overwhelmed with elastic scattered photons or fluorescent photons. Although lock-in detection is highly successful in recovering a weak electrical ac signal from large noise background, phase sensitive detection (PSD) of a weak optical signal at the photon counting level often requires special care. Recently, a computer-based PSD scheme had been developed for the detection of an extremely weak optical signal with a conventional photon counting hardware [1]. The functionality of the methodology, however, has not yet been verified in a practical application such as Raman spectroscopic and imaging system and the characteristics of the methodology have not yet been fully explored.

Ferroelectric liquid crystals (FLC) can be used for flat panel displays (FPD) with advantages of fast response, bistability, and wide viewing angle [2,3]. However, the detailed knowledge of FLC alignment during the field-induced reorientation process is still lacking. Due to the interests of both fundamental and applied research, many efforts have been made to investigate the electric-field- induced reorientation behavior of FLC. In this report, we employed the PSD technique with Raman microscopy to probe the reorientation

*Address correspondence to this author at the Department of Photonics, Chiao Tung University, Taiwan, Republic of China; Tel: +886-3-5731975; Fax: +886-3-5716631; E-mail: jyhuang@faculty.nctu.edu.tw dynamics of a surface stabilized ferroelectric liquid crystals (SSFLC) cell. The phase-sensitive Raman imaging scheme yields rich information about the electro-optic active species that are closely related to the applications of SSFLC. Theoretical model of the PSD Raman signal from a molecular normal mode was presented to illustrate the underlying principle.

This paper is organized as follows: in Sec. 2, we present the theoretical background of the PSD Raman signal from a molecular normal mode. The experimental setup is depicted in Sec. 3. In Sec. 4, experimental results are presented to show the functionalities of the method and finally conclusion is drawn.

\section{THEORETICAL BACKGROUND AND EXPERI- MENTAL METHODS}

\subsection{Raman Scattering from Surface-Stabilized FLC}

Fig. (1) shows the schematic of a SSFLC cell in a Raman scattering measurement. Here $\mathrm{Z}$ represents the rubbing direction, which is also the layer normal of the smectic layers of SSFLC. The direction of FLC molecules can be switched by an applied electric field $\boldsymbol{E}$. The polarization angle of the incident light field $\boldsymbol{E}_{\mathbf{L}}$ is taken to be zero when the polarization direction coincides with the rubbing direction. $\boldsymbol{M}$ on the right diagram, which tilts from the molecular long axis $\xi$ with an angle of $\beta$ and rotates about the $\xi$-axis by $\gamma$, denotes the molecular normal mode responsible for the Raman scattering.

The number of Raman scattered photons from FLC molecules is proportional to the square of projected Raman polarizability $\alpha_{i j}$. Assuming $\alpha_{i j}$ of a normal mode with $\beta=0$ to be a tensor with single dominating component $\alpha_{M M}$, the $Y$-polarized Raman signal $\left(\Phi=90^{\circ}\right)$ excited by a $Y$ polarized optical field can therefore be expressed as

$$
R_{Y \rightarrow Y}(v, t)=R_{0} \sin ^{4} \theta\{3 \sin \phi(t)+\sin [3 \phi(t)]\}^{4}
$$




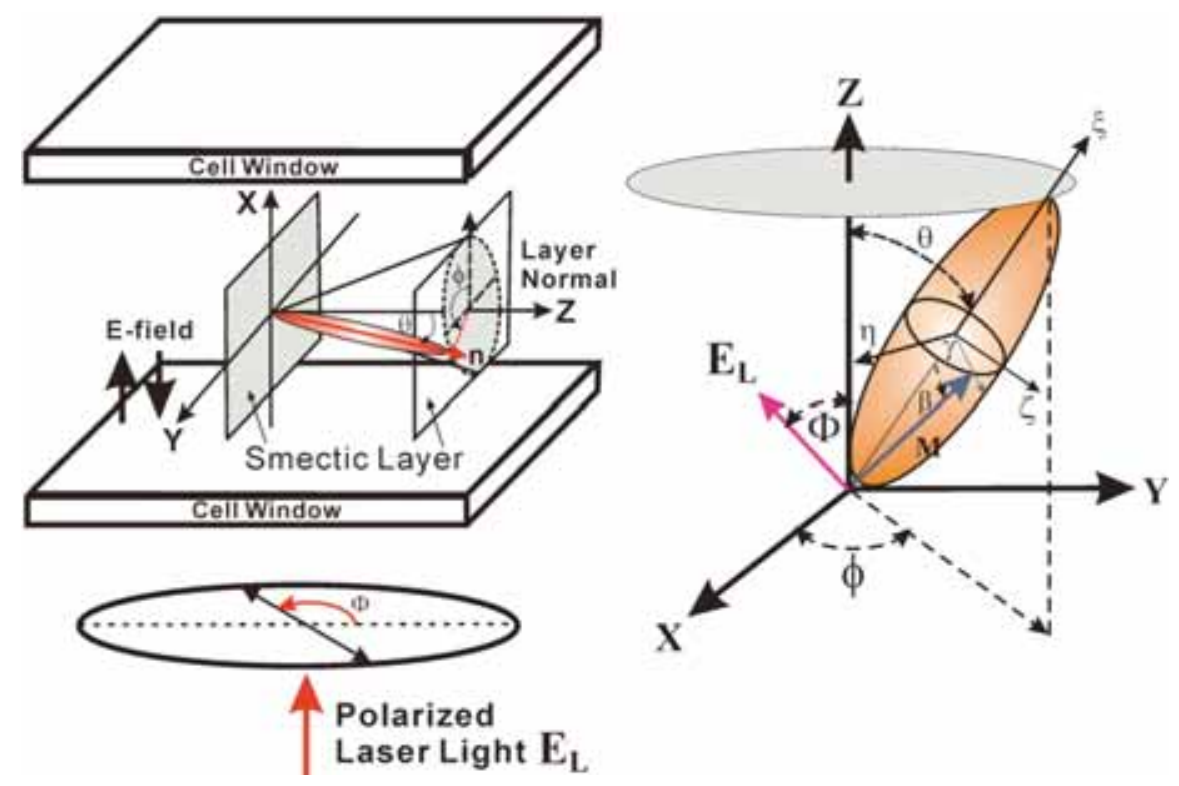

Fig. (1). Schematic of a SSFLC cell in a Raman scattering setup: $\boldsymbol{Z}$ denotes the rubbing direction, which also the layer normal of the smectic layers of SSFLC. $\boldsymbol{E}$ is the direction of the applied electric field. The polarization angle of the incident polarized light $\boldsymbol{E}_{\mathbf{L}}$ is taken to be zero when its polarization direction coincides with the rubbing direction. $\boldsymbol{M}$ on the right diagram denotes the molecular normal mode, which tilts from the molecular long axis $\xi$ with an angle of $\beta$ and rotates about the $\xi$-axis by $\gamma$.

Similarly, the Z-polarized Raman signal $\left(\Phi=0^{\circ}\right)$ excited by a Y-polarized optical field becomes

$R_{Y \rightarrow Z}(v, t)=R_{0} \sin ^{2}(2 \theta) \sin ^{2} \phi(t)\left[2+\cos ^{2}[2 \phi(t)]\right\}^{2}$

Notice that the azimuthal angle $\phi(t)$ of FLC molecules on the $\mathrm{SmC}^{*}$ cone can be electrically controlled with a sinusoidal waveform of frequency $\Omega$.

\subsection{Phase-Sensitive Detection}

The analytical procedure of PSD at a given wave number $v$ involves a multiplication of the time-resolved Raman spectrum $R(v, t)$ with $\cos [\Omega t+\Psi]$ followed by a normalized integration over a period of $T$

$\bar{R}_{\Psi}(v, \Omega)=\frac{2}{T} \int_{0}^{T} R(v, t) \cos [\Omega t+\Psi] d t$

This procedure produces a phase-resolved spectrum $\bar{R}_{\psi}(v, \Omega)$ associated with a modulation frequency $\Omega$ and phase setting $\Psi$. Applying Equation (3) to all wavenumber $v$ of the spectrum leads to a data vector. The special cases of $\bar{R}_{\Psi}(v, \Omega)$ with $\Psi=0^{\circ}$ and $\Psi=90^{\circ}$ are equivalent to the in-phase $\bar{R}(v, \Omega) \cos [\Delta(v, \Omega)] \quad$ and out-of-phase components $\bar{R}(v, \Omega) \sin [\Delta(v, \Omega)]$ of a lock-in detection scheme:

$$
\begin{aligned}
& \bar{R}(v, \Omega) \cos [\Delta(v, \Omega)]=\bar{R}_{\Psi=0^{0}}(v, \Omega)=\frac{2}{T} \int_{0}^{T} R(v, t) \cos [\Omega t] d t \\
& \bar{R}(v, \Omega) \sin [\Delta(v, \Omega)]=\bar{R}_{\Psi=90^{0}}(v, \Omega)=\frac{2}{T} \int_{0}^{T} R(v, t) \sin [\Omega t] d t
\end{aligned}
$$

Here $\bar{R}(v, \Omega)$ and $\Delta(v, \Omega)$ denote the modulation amplitude and the corresponding phase shift of the PSD Raman signal. These are the main parameters important for the interpretation of a PSD experiment and can be determined from the measured phase-resolved spectra $\bar{R}_{\Psi=0^{0}}(v, \Omega)$ and $\bar{R}_{\Psi=90^{0}}(v, \Omega)$ by using

$$
\begin{aligned}
& \bar{R}(v, \Omega)=\sqrt{\left[\bar{R}_{\Psi=0^{0}}(v, \Omega)\right]^{2}+\left[\bar{R}_{\Psi=90^{0}}(v, \Omega)\right]^{2}} \\
& \sin [\Delta(v, \Omega)]=\bar{R}_{\Psi=90^{0}}(v, \Omega) / \bar{R}(v, \Omega) \\
& \cos [\Delta(v, \Omega)]=\bar{R}_{\Psi=0^{0}}(v, \Omega) / \bar{R}(v, \Omega)
\end{aligned}
$$

\subsection{Experimental Methods}

The laser used in our Raman imaging apparatus is a 50$\mathrm{mW} \mathrm{CW}$ diode-pumped solid state laser operating at $532 \mathrm{~nm}$. The laser was weakly focused with a lens to yield an elliptical spot of $90 \mu \mathrm{m} \times 60 \mu \mathrm{m}$ on the sample at an incident angle of $45^{\circ}$. The SSFLC cell was driven with a sinusoidal electric waveform. The Raman scattering photons were filtered with a Raman notch filter and a spectrograph and then detected with a cooled photomultiplier tube. The photocurrent was processed by a single-photon counting module. The lock-in amplitude and phase were retrieved in real time with a software-based phase-sensitive single-photon counting scheme [1].

The SSFLC cells used for this study consist of two glass plates coated with indium tin oxide (ITO) and polyimide alignment layers as depicted in Fig. (1). The substrates were separated by $2 \mu \mathrm{m}$-thick spacers to maintain a cell gap close to the half-wave thickness [4].

Felix 017/100 from Clariant Inc. was used in view of its excellent device application properties. To prepare $n c-\mathrm{ZnO}$ doped SSFLC, an appropriate amount of $n c-\mathrm{ZnO}$ powder was dispersed in pure Felix 017/100 to 1.0 weight percent [5]. The resulting homogeneous mixture was filled into the test cells and then cooled slowly to $35^{\circ} \mathrm{C}$ to maintain a stable SmC* phase. All studies were performed on SSFLC cells with single domain. 


\section{RESULTS}

\subsection{SSFLC Cell with Pure Felix 017/100}

A SSFLC cell with pure Felix 017/100 was investigated first with Raman spectroscopy without applying an electric modulation field. The cell was excited with an $s$-polarized ( $Y$ axis) 532-nm laser beam. The Raman spectrum is presented in Fig. (2a). The observed Raman peaks at $1610 \mathrm{~cm}^{-1}, 1505 \mathrm{~cm}^{-1}$, and $1326 \mathrm{~cm}^{-1}$ can be attributed to the $\mathrm{C}=\mathrm{C}$ stretching modes of the phenyl rings, the $1446 \mathrm{~cm}^{-1}$ peak to the $\mathrm{C}=\mathrm{C}$ stretch of pyrimidine ring, and the $1118 \mathrm{~cm}^{-1}$ to the $\mathrm{C}-\mathrm{O}-\mathrm{C}$ motion [6].
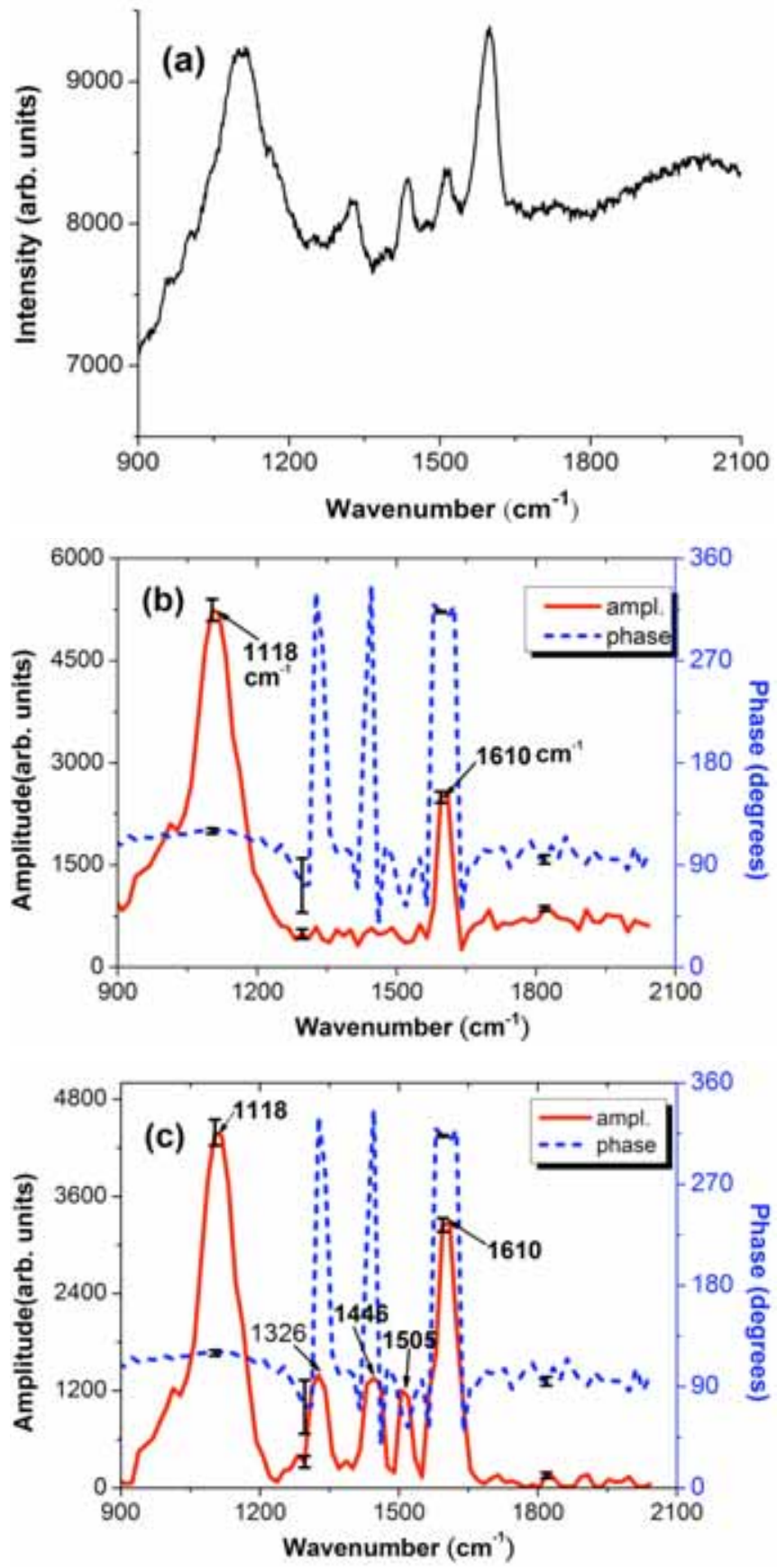

Fig. (2). (a) Raman spectrum of a SSFLC cell. (b) The amplitude (solid curve) and phase (dashed line) profiles of a PSD Raman spectrum $\bar{R}_{\Psi}(v, \Omega)$ with a modulation frequency $\Omega=250 \mathrm{~Hz}$. (c)

The corresponding PSD amplitude (solid curve) after removing the nonresonant background.
When a photon-counting lock-in detection scheme was employed, the amplitude and phase of the PSD Raman signal from the SSFLC cell driven with a sinusoidal waveform of $\Omega=250 \mathrm{~Hz}$ can be obtained. The results are presented in Fig. (2b). Although the nonresonant background had been effectively suppressed by the lock-in detection scheme, a trace of residual nonresonant background is still detectable, which most likely originates from light scattering by the fieldinduced refractive index variation. The phase of the nonresonant signal, measurable at the spectral region far from any resonance, was found to be about $90^{\circ}$. The three Raman peaks at $1326 \mathrm{~cm}^{-1}, 1446 \mathrm{~cm}^{-1}$, and $1505 \mathrm{~cm}^{-1}$ disappear because their PSD amplitudes are comparable to that of the nonresonant background whereas with $180^{\circ}$ phase difference. By using the phase value, we can remove the residual nonresonant background from the PSD spectrum and the result is presented in Fig. (2c) with the solid curve. The $90^{\circ}$ phase angle of the nonresonant signal causes the measured phase of the Raman peaks to vary from $60^{\circ}$ (at $1505 \mathrm{~cm}^{-1}$ ), $\Delta \sim 120^{\circ}$ (at $1118 \mathrm{~cm}^{-1}$ ), $180^{\circ}$ (at both $1446 \mathrm{~cm}^{-1}$ and $1326 \mathrm{~cm}^{-1}$ ), to $\Delta \sim 320^{\circ}$ (at $1610 \mathrm{~cm}^{-1}$ ). The phase variation most likely originates from the different responses of inter- and intramolecular functional groups to an applied electric field and therefore carries the dynamic structural information of electro-optic active species in a complex thin film.

In the frequency domain, an SSFLC cell driven by a sinusoidal waveform shall exhibit a variation of the Raman scattered light and a phase shift between the scattered light and the electric field. The relationship between the driving field and the PSD Raman response can be described by a transfer function $H(\Omega)$

$\frac{\Delta \bar{R}_{\psi}(v, \Omega)}{\bar{R}_{0}(v)}=H(\Omega)\left|\frac{\Delta E(\Omega)}{E_{0}}\right|=\frac{1}{1-L \Omega^{2}+i \Omega \tau}\left|\frac{\Delta E(\Omega)}{E_{0}}\right|$

Here $E_{0}$ is an appropriate normalization parameter and $\Delta E(\Omega)$ denotes the ac component of the driving electric field; while $\bar{R}_{0}(v)$ is the dc component of the Raman peak, and $\Delta \bar{R}_{\Psi}(v, \Omega)$ the amplitude of PSD Raman peak.

An optical transmittance image in an area of 1500 $\mu \mathrm{m} \times 1500 \mu \mathrm{m}$ of the film is shown in Fig. (3a). Two defects of SSFLC can be observed in this region. The corresponding 2D distributions of the amplitude and the phase of the 1610$\mathrm{cm}^{-1}$ Raman PSD peak with $\Omega=250 \mathrm{~Hz}$ are presented in Fig. $(3 \mathbf{b}, \mathbf{c})$. Both the dc Raman peak (not shown) and the PSD signal amplitude at $1610 \mathrm{~cm}^{-1}$ are very weak inside the defects, indicating the defects to be a void without FLC. To further unveil the characteristics of the PSD Raman imaging technique, we focused on two locations marked with $\mathbf{p} 1$ and p2 in Fig. (3b, c) for further examination. The site $\mathbf{1}$ was chosen to show the intrinsic characteristics of the SSFLC cell and the site 2 reveals the influences from the rim of a void defect.

The experimental results of a flexible C-O-C motion at $1118 \mathrm{~cm}^{-1}$ measured at site $\mathbf{1}$ as a function of modulation frequency $\Omega$ are presented in Fig. (4a). The measured phases of the PSD Raman signal are presented with open symbols and the measured magnitudes are shown with filled symbols. Since the transfer function of PSD phase can only be affected by the modulation, we therefore first determine the 

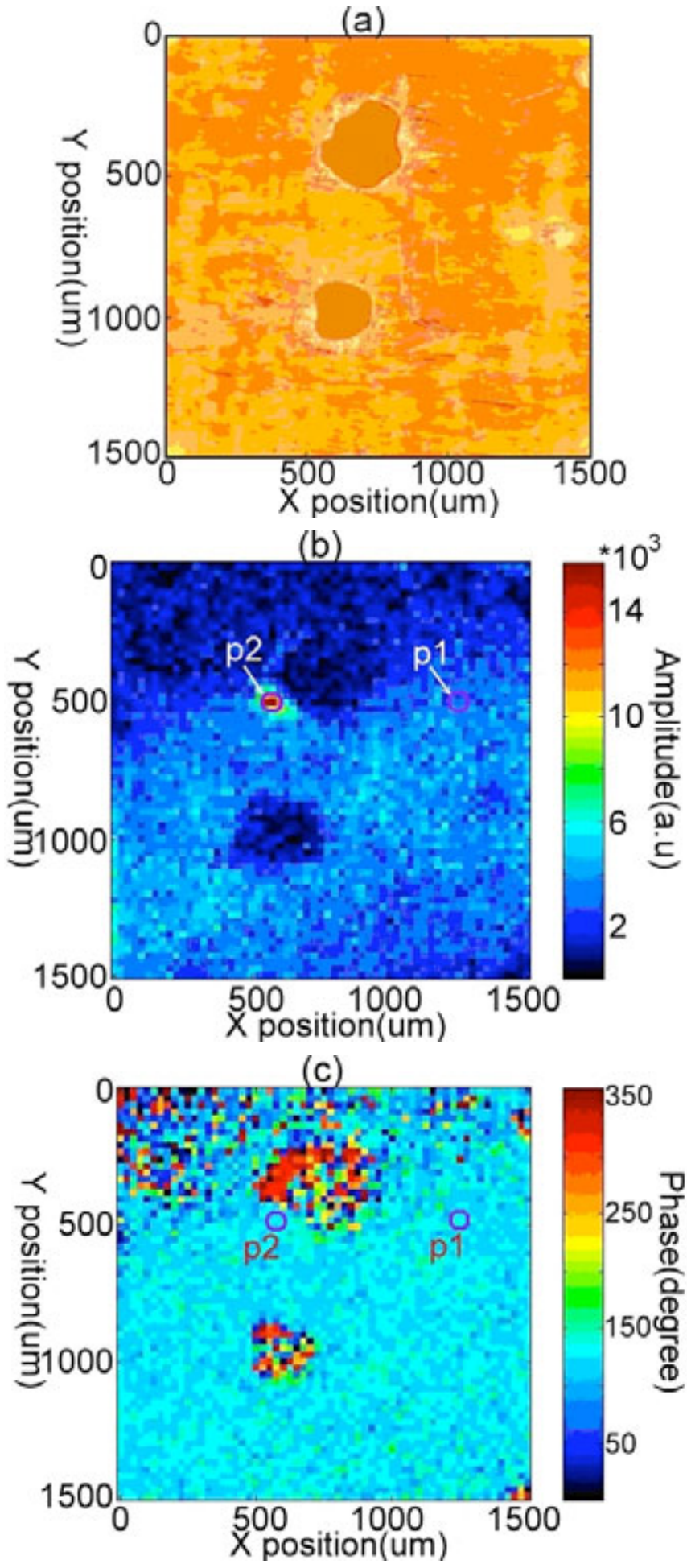

Fig. (3). (a) The optical transmittance image of a Felix 017/100 SSFLC cell, and the 2D distributions of (b) the amplitude, and (c) the phase of the PSD Raman peak at $1609 \mathrm{~cm}^{-1}$ with a modulating frequency $\Omega=250 \mathrm{~Hz}$.

phase relaxation time from a fit of the measured phase of the PSD Raman signal at $1118-\mathrm{cm}^{-1}$ to Equation (6). The fitting yields a relaxation time of $6.27 \times 10^{-3} \mathrm{sec}$. Unlike the phase relaxation process, the magnitude of the PSD Raman signal as indicated in Equations (1) and (2) can vary with modulation and the alignment of FLC molecules. Since FLC molecules could be switched to form a less ordered structure with a high-frequency driving field, a modulus transfer function with wider $3-\mathrm{dB}$ bandwidth than that revealed by the phase transfer function shall be obtained. This is what we had observed in Fig. (4a), where the modulus transfer function of the PSD Raman response can be fitted with $\tau=1.54 \times 10^{-3} \mathrm{sec}$ (solid curve), which is 4 times shorter than that of the phase relaxation time given above. In the following, we will use the reduction factor from the phase relaxation time to the magnitude relaxation time to unveil the high-frequencydriving induced disordering in the FLC alignment.
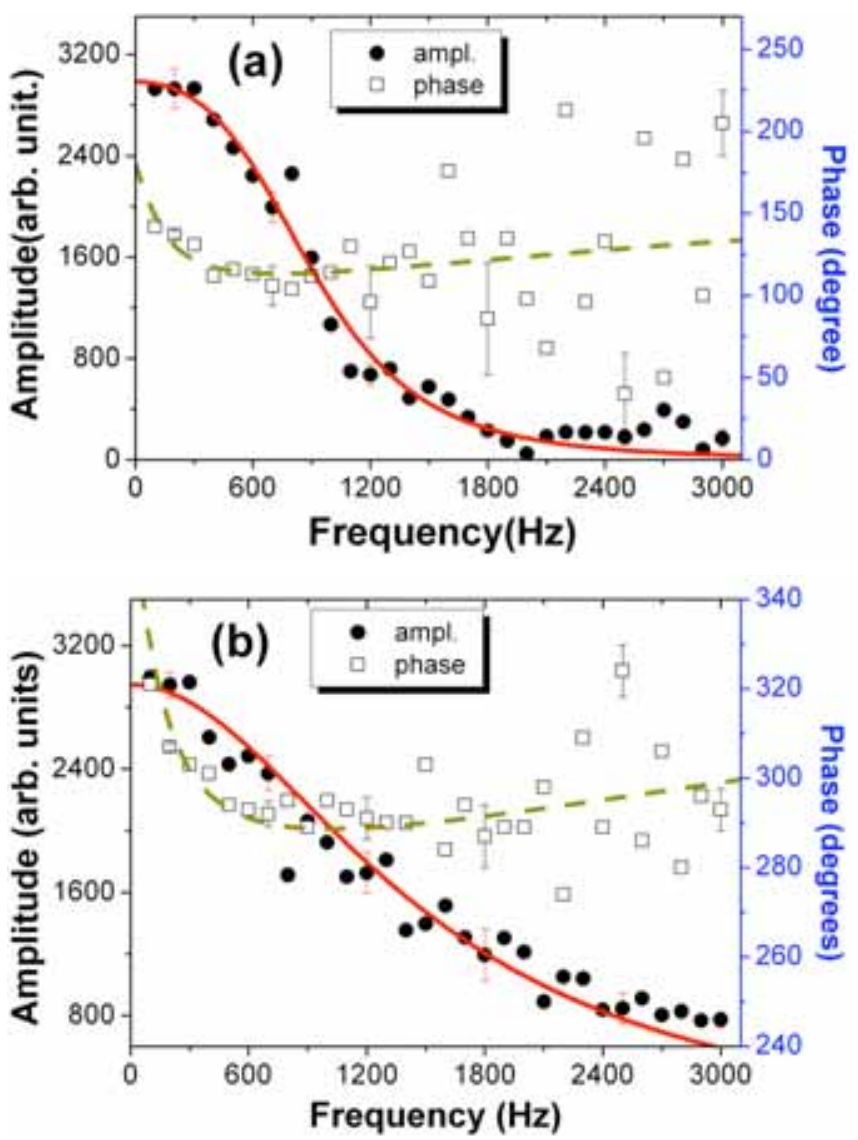

Fig. (4). The amplitude (filled circles) and phase (open squares) of a phase-resolved Raman peak (a) at $1118 \mathrm{~cm}^{-1}$ and (b) at $1610 \mathrm{~cm}^{-1}$ at the site-1 are presented as a function of modulation frequency $\Omega$ in a pure SSFLC cell. The lines are the fitting to Equation (6).

The experimental result of the $1610 \mathrm{~cm}^{-1}$-peak (the $\mathrm{C}=\mathrm{C}$ stretching mode of the core part of FLC molecules) measured at the site 1 as a function of $\Omega$ is presented in Fig. (4b). The relaxation time of the PSD Raman magnitude was determined to be $6.7 \times 10^{-4} \mathrm{sec}$, which is about 8.7 times smaller than the corresponding phase relaxation time $5.8 \times 10^{-3} \mathrm{sec}$. The result indicates that a normal mode with rigid nature appears to have a more distinctive reduction effect.

The modulus transfer function of the PSD Raman response at $1118 \mathrm{~cm}^{-1}$ at the site 2 yields a relaxation time of $6.05 \times 10^{-3} \mathrm{sec}$. Comparing to the corresponding phase relaxation time $\left(1.23 \times 10^{-3} \mathrm{sec}\right)$, the reduction factor is slightly increased from 4 at the site 1 to 4.9 at the site 2 . The relaxation time of the $1610 \mathrm{~cm}^{-1}$-peak at the position 2 decreases from $5.53 \times 10^{-3} \mathrm{sec}$ (the phase relaxation time) to $5.44 \times 10^{-4} \mathrm{sec}$ (the amplitude relaxation time). The reduction factor increases from 8.7 at the site $\mathbf{1}$ to 10.2 at the site $\mathbf{2}$, supporting 
the notion that the reduction factor for a normal mode with rigid nature is more sensitive to environmental conditions, such as the rim of a void on the field-induced reorientation dynamics of FLC molecules.

\subsection{SSFLC Cell with Felix 017/100 Doped with $n c-\mathrm{ZnO}$}

In our previous study, we discovered that the electrooptical response of FLC can be improved by doping with zinc oxide nanocrystals $(n c-\mathrm{ZnO})$ [5]. The Raman microscopy with photon-counting lock-in detection can be used to yield an insight into the electro-optical switching SSFLC film with $n c-\mathrm{ZnO}$ doping.

The optical transmittance image of a SSFLC cell of Felix $017 / 100$ doped with $n c-\mathrm{ZnO}$ is shown in Fig. (5a). The 2D distributions of the amplitude and the phase of the PSD Raman signal at $1610 \mathrm{~cm}^{-1}$ with a modulation frequency $250 \mathrm{~Hz}$ are presented in Fig. $(\mathbf{5 b}, \mathbf{c})$. The optical image shown in Fig. (5a) indicates the lower right portion of the region to be different from the rest part. Fig. (5b) reveals that FLC molecules in this region generate weaker PSD Raman signals, indicating that the FLC molecules are somewhat disordered to yield a weaker electro-optical response. Two sites labeled with p1 and p2 were chosen for further investigation. Position $\mathbf{p 1}$ is a region with typical SSFLC properties, while the position $\mathbf{p 2}$ represents an alignment structure of enhanced electro-optical response.

The dc Raman spectrum of the $n c-\mathrm{ZnO}$ doped SSFLC cell excited with a $Y$-polarized laser beam at $532 \mathrm{~nm}$ is presented in Fig. (6a). The Raman spectrum is very similar to that shown in Fig. (2a), except that the $1118 \mathrm{~cm}^{-1}$ peak from the $\mathrm{C}-\mathrm{O}-\mathrm{C}$ motion is weaker. The amplitude and phase of the PSD Raman spectrum with a sinusoidal waveform of $\Omega=250$ $\mathrm{Hz}$ are presented in Fig. (6b). The phase of the nonresonant background signal was found to be about $330^{\circ}$. By using the phase angle, we can remove the residual nonresonant background from the spectrum. The resulting PSD Raman amplitudes are shown with solid curve in Fig. (6c). The phase of the PSD Raman signal exhibits $\Delta \sim 330^{\circ}$ at $1610 \mathrm{~cm}^{-1}$, and $\Delta \sim 140^{\circ}$ at $1118 \mathrm{~cm}^{-1}$, which are similar to that of the undoped SSFLC film shown in Fig. (2b). The phase of the $\mathrm{C}=\mathrm{C}$ stretching PSD Raman signal was found to be about $330^{\circ}$, which is in phase with the nonresonant signal. As pointed out above, the nonresonant background is most likely to originate from light scattering from the field-induced refractive index variation. The zero phase difference between the nonresonant signal and the $\mathrm{C}=\mathrm{C}$ stretching peak indicates that doping SSFLC with $n c-\mathrm{ZnO}$ results in a more organized field-induced reorientation process with fast response to an applied field (with smaller phase lag).

The measured transfer function of the Raman peak at $1610 \mathrm{~cm}^{-1}$ from the positions $\mathbf{p 1}$ and $\mathbf{p 2}$ are presented in Fig. (7a, b). The solid curves are the fitting to Equation (6). The relaxation time deduced from the measured PSD magnitude at $1610 \mathrm{~cm}^{-1}$ at the position $\mathbf{p 1}$ is $9.89 \times 10^{-4} \mathrm{sec}$, which is about 5 times shorter than $4.98 \times 10^{-3} \mathrm{sec}$ of the phase relaxation time. At the position $\mathbf{p 2}$, the PSD amplitude relaxation time becomes $6.81 \times 10^{-4} \mathrm{sec}$, which is about 3.5 times shorter than the corresponding phase relaxation time $2.36 \times 10^{-3}$. Our results therefore indicate that the field-induced reorientation of the $n c-\mathrm{ZnO}$ doped SSFLC film at the position $\mathbf{p} 2$ is more organized and less sensitive to the high-frequency-driving induced disordering.
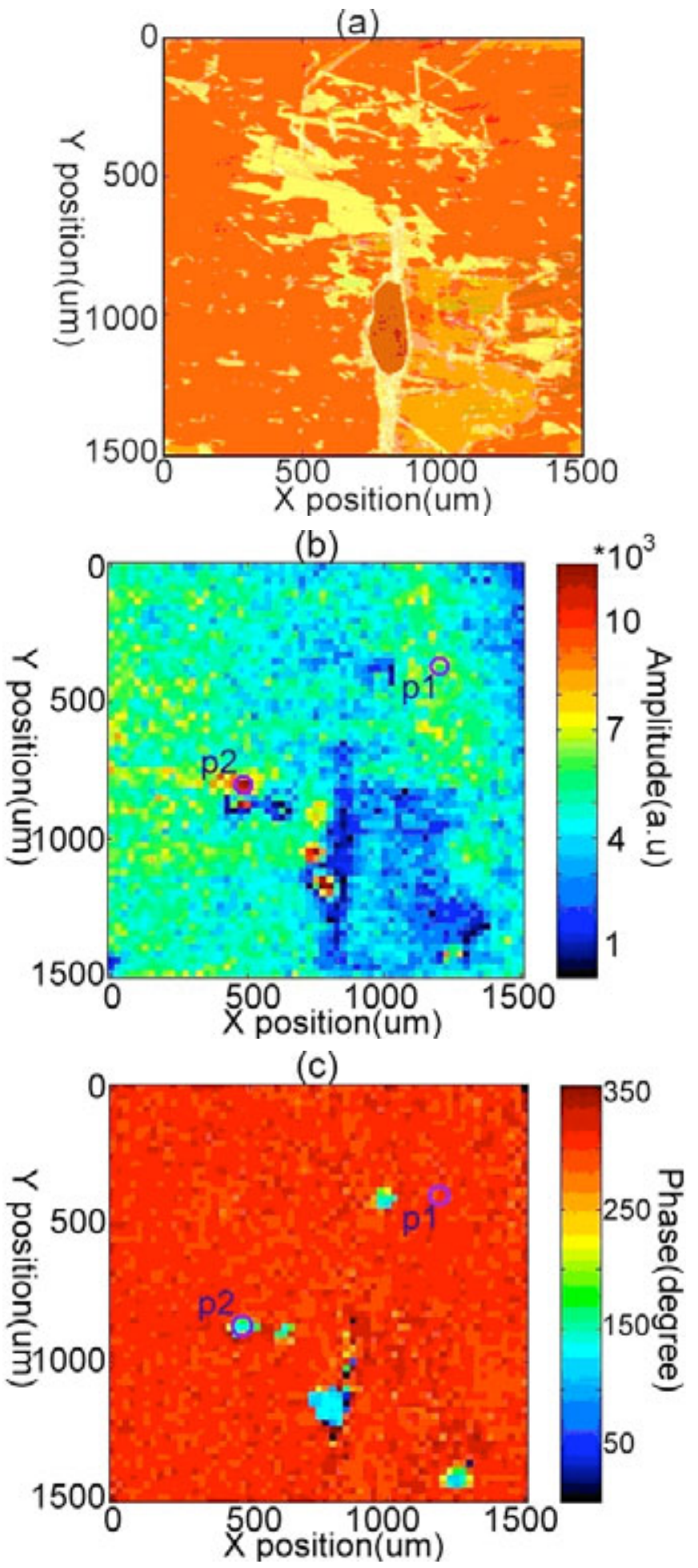

Fig. (5). (a) The optical image of a scanned region on a Felix $017 / 100$ SSFLC cell doped with $n c-\mathrm{ZnO}$, and the 2D distributions of (b) the amplitude, and (c) the phase of the lock-in detected Raman peak at $1609 \mathrm{~cm}^{-1}$ with a modulated frequency $\Omega=250 \mathrm{~Hz}$. 

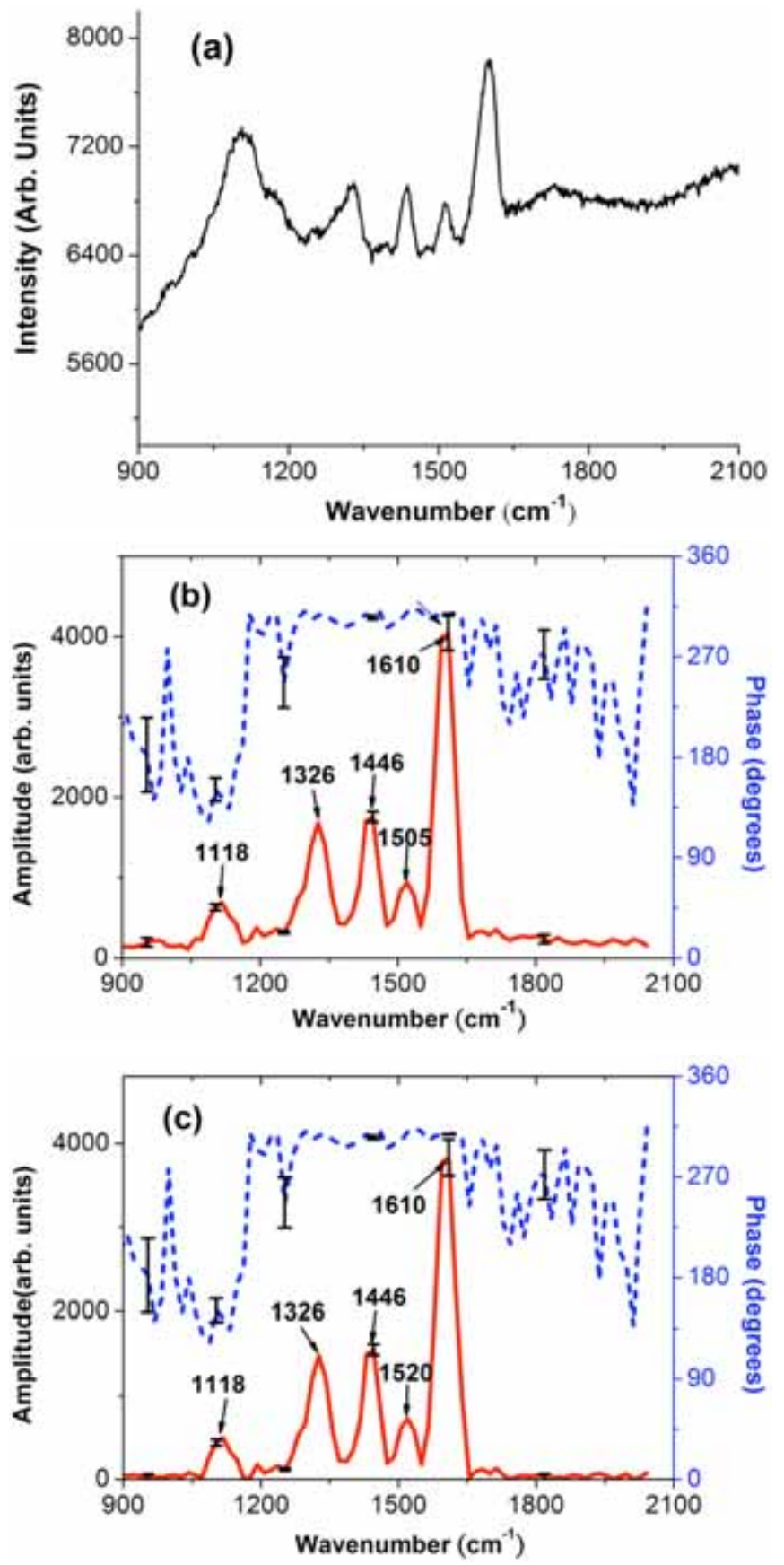

Fig. (6). (a) DC Raman spectrum of a SSFLC cell doped with $n c$ $\mathrm{ZnO}$ excited at $532 \mathrm{~nm}$. (b) The amplitude (solid curve) and phase (dashed line) profiles of a lock-in detected Raman spectrum $\bar{R}_{\Psi_{p S D}}(v, \Omega)$ with a modulation frequency $\Omega=250 \mathrm{~Hz}$ measured at the position p1 shown in Fig. (5b). (c) The corresponding lock-in detected amplitude (solid curve) after removing the nonresonant background.

\section{DISCUSSION AND CONCLUSIONS}

Dielectric dispersion measurements on spherical CdSe nanocrystallites reported in [7] suggest that these nanocrystals have an internal electrostatic polarization resulting from a dipole moment that scales with the particle size. The dipole moment is only mildly screened by passivation layer. Similar to $\mathrm{CdSe}$ quantum dots, $\mathrm{ZnO}$ possesses wurtzite structure that
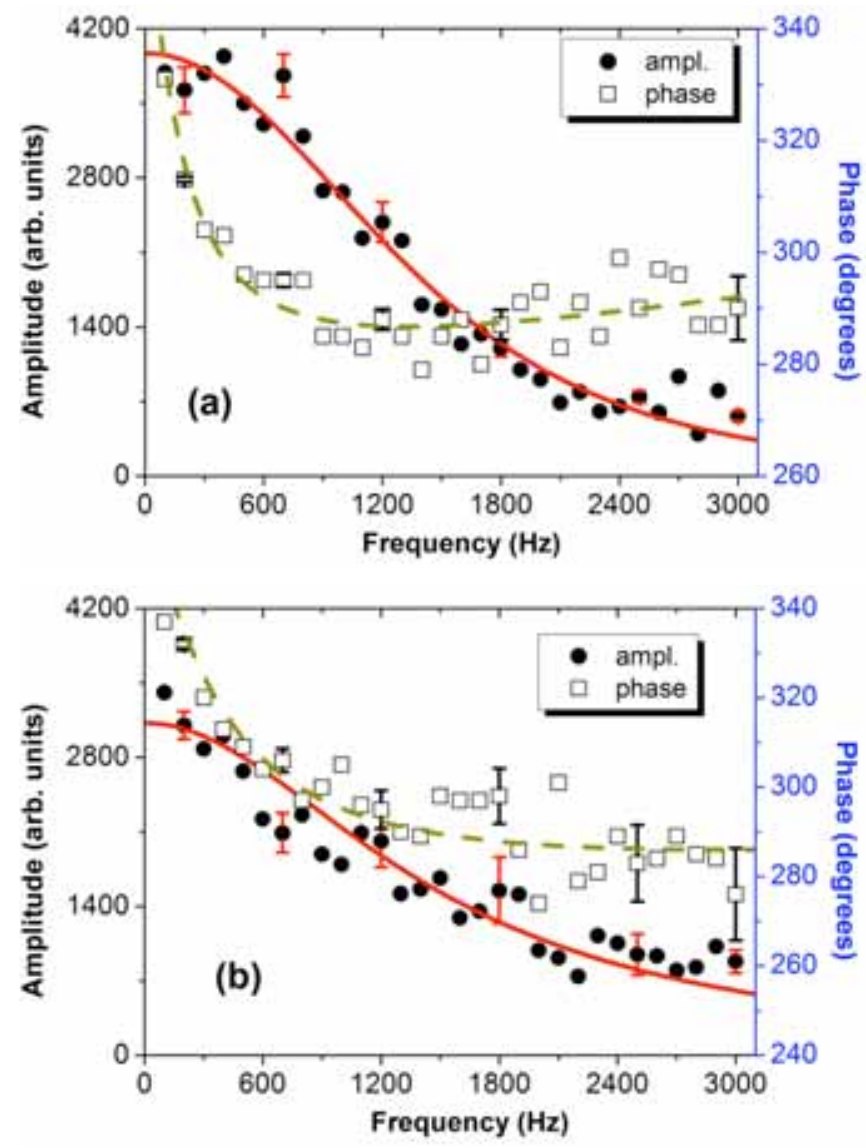

Fig. (7). The amplitude (filled circles) and phase (open squares) of a phase-resolved $1609 \mathrm{~cm}^{-1}$-Raman peak of a $n c-\mathrm{ZnO}$ doped SSFLC cell measured at (a) the position $\mathbf{p} 1$ and (b) the position $\mathbf{p} 2$ are presented as a function of modulation frequency $\Omega$. The lines are the fitting to Equation (6).

allows the $\mathrm{ZnO}$ nanoparticles with a diameter of $2 R=3.5 \mathrm{~nm}$ to carry a dipole moment of $\mu_{Z n O}=50$ Debye. The particles being nano-size objects behave like a molecular dopant. At one weight percent of $n c-\mathrm{ZnO}$ in FLC, there are about $n_{F L C}=$ 15000 FLC molecules surrounding one single $\mathrm{ZnO}$ nanoparticle within a spherical space of $R_{\max }=14 \mathrm{~nm}$. For FLC molecules with polar $\mathrm{C}=\mathrm{O}$ groups, each $\mathrm{ZnO}$ nanodot can exert a dipolar interaction on surrounding FLC molecules. We can estimate the thermally-averaged energy density of the dipoledipole interaction between the $\mathrm{ZnO}$ nano dot and FLC dipoles by using [8]:

$U_{a}^{Z n O-L C}=-\frac{2 \mu_{C O}^{2} \mu_{Z n O}^{2} n_{F L C} N_{Z n O}}{3\left(4 \pi \varepsilon_{0}\right)^{2} k_{B} T R_{0}^{3} R_{\max }^{3}}$

By taking $N_{\mathrm{ZnO}}=1.4 \times 10^{23} \mathrm{~m}^{-3}, \quad \mu_{C O}=1.5$ Debye, $\mu_{\mathrm{ZnO}}=50$ Debye, and $k_{B} T=4 \times 10^{-21} \mathrm{~J}$, we found $U_{a}^{\mathrm{ZnO}-F L C} \simeq-1000 \mathrm{~J} \cdot \mathrm{m}^{-3}$. The $n c-\mathrm{ZnO}$ doping induced energy decrease has the same order as the surface anchoring energy density near an alignment surface and shall be able to render the FLC aligning structure in more unison.

In summary, we had applied photon counting phasesensitive detected (PSD) Raman imaging technique to inves- 
tigate the modulation dynamics of surface stabilized ferroelectric liquid crystal with and without doping of $\mathrm{ZnO}$ nanocrystals. The technique not only effectively suppresses the nonresonant background of Raman spectrum, but also yields insightful information about the PSD amplitude and phase of specific Raman peak. The reduction factor from the phase relaxation time to the amplitude relaxation time was found to be sensitive to the disordering effect of the FLC alignment from the high-frequency driving condition. The fieldinduced reorientation dynamics of the $n c-\mathrm{ZnO}$ doped SSFLC film is generally more organized and therefore less sensitive to the high-frequency driving induced disordering. The effect was attributed to originate from $\mathrm{ZnO}$ nano dots that could act as an effective molecular binder by tying together surrounding FLC dipolar species.

\section{ACKNOWLEDGEMENT}

We acknowledge the financial support from the National Science Council of the Republic of China under grant NSC 95-2112-M-009-031.

\section{REFERENCES}

[1] Braun D, Libchaber A. Computer-based photon-counting lock-in for phase detection at the shot-noise limit. Opt Lett 2002; 27: 1418-3.

[2] Clark NA, Lagerwall ST. Submicrosecond bistable electro-optic switching in liquid crystals. Appl Phys Lett 1980; 36: 899-3.

[3] Lagerwall ST. Ferroelectric and antiferroelectric liquid crystals, Weinheim: Wiley-VCH; 1999.

[4] Krueger M, Giesselmann F. Dielectric spectroscopy of de Vriestype smectic- $A^{*}$-smectic- $C^{*}$ transitions. Phys Rev E Stat Nonlin Soft Matter Phys 2005; 71: 041704-8.

[5] Huang JY, Li LS, Chen MC. Probing molecular binding effect from zinc oxide nanocrystal doping in surface-stabilized ferroelectric liquid crystal with two-dimensional infrared correlation technique. J Phys Chem C 2008; 112: 5410-5.

[6] Huang JY, Shih WT. Probing field-induced submolecular motions in a ferroelectric liquid crystal mixture with time-resolved twodimensional infrared spectroscopy. J Phys Condens Matter 2006; 18: 7593-10.

[7] Shim M, Guyot-Sionnest P. Permanent dipole moment and charges in colloidal semiconductor quantum dots. J Chem Phys 1999; 111: 6955-9.

[8] Li F, Buchnev O, Cheon CI, et al. Orientational coupling amplification in ferroelectric nematic colloids. Phys Rev Lett 2006; 97: 147801-4.

(c) Wang and Huang; Licensee Bentham Open.

This is an open access article licensed under the terms of the Creative Commons Attribution Non-Commercial License (http://creativecommons.org/licenses/by$\mathrm{nc} / 3.0 /$ ) which permits unrestricted, non-commercial use, distribution and reproduction in any medium, provided the work is properly cited. 